\title{
Fabrication of long hexagonal micro-lens array by applying gray-scale lithography in micro-replication process
}

\author{
Jauh-Jung Yang ${ }^{\text {a,1 }}$, Yunn-Shiuan Liao ${ }^{\mathrm{a}, *}$, Chi-Feng Chen ${ }^{\mathrm{b}, 2}$ \\ a Department of Mechanical Engineering, National Taiwan University, Taipei, Taiwan, ROC \\ ${ }^{\mathrm{b}}$ Department of Mechanical Engineering and Institute of Opto-Mechatronics Engineering, National Central University, Chung-li, Taiwan, ROC
}

Received 27 March 2006; received in revised form 25 July 2006; accepted 7 September 2006

\begin{abstract}
A hexagonal micro-lens (HML) array with long aspect ratio of 3.3:1 designed for beam shaping was fabricated by applying gray-scale lithography in micro-replication process. The gray-scale lithography process was employed in this study to obtain unity curvature and to maximize filling factor of the lens array. Precision micro-replication process was applied to duplicate HML on a flexible polyethylene terphthalate (PET) substrate by UV-forming process. The geometry character of obtained HML was inspected and the shape of HML is very close to the designed specification. The optical performance was also investigated. It was found that the incident Gauss beam was diversified differently at short and long axis. The beam full widths at the half maximum (FWHM) at short axis and long axis directions through the HML are $0.61 \mu \mathrm{m}$ and $0.25 \mu \mathrm{m}$, respectively. Hence, the beam divergent ratio at long and short axis of $2.44: 1 \mathrm{was}$ achieved.
\end{abstract}

(C) 2006 Elsevier B.V. All rights reserved.

Keywords: Gray-tone lithography; Hexagonal micro-lens array; Micro-fabrication technology; Micro-replication

\section{Introduction}

Refractive micro-lens has been in increasing demand for various optical applications $[1,2]$ specifically in imaging systems [3], digital display systems [4], digital optical processors [5], and optical interconnection [6], etc. It possesses many advanced optical features such as low wavelength sensitivity, large numerical apertures, high light efficiency and is expected to play a major role in miniature optical system in the future. The majority of the researches regarding refractive micro-lens conducted recently are related to a symmetric shape such as round, square and regular hexagonal lenses [7]. But in certain applications e.g. liquid-crystal display, projector, light emitting diode lighting, etc.,

\footnotetext{
* Corresponding author. Tel.: +8862 23626431; fax: +886223631755.

E-mail addresses: d93522029@ntu.edu.tw (J.-J. Yang), liaoys@ntu. edu.tw (Y.-S. Liao), ccf@cc.ncu.edu.tw (C.-F. Chen).

${ }^{1}$ Tel.: +886 283693441 ; fax: +886223661347

${ }^{2}$ Tel.: +886 3 4267308; fax: +88634254501.
}

there are two important factors needed to be considered. One is asymmetrically shaped of lens which offers more flexibility to the optical designer to control the illumination patterns. The other is filling factor which influences strongly light utilization. The filling factor is defined as the percentage of lens area to the total area. To maximize the light collection, the lenses should be gapless as much as possible to close to $100 \%$ of total area. Hence, it is essential to develop a precision and efficient approach to fabricate asymmetrical shape lens array with near $100 \%$ filling factor.

Regarding fabrication methods of the refractive microlens profile, many approaches have been reported. The most commonly used method to form spherical microlenses is the thermal reflow technique. By this method, the micro-cylinders made of photoresist are firstly produced by lithography process and are subsequently heated to a temperature above the $T_{\mathrm{g}}$ (glass transition temperature) of the photoresist. Surface tension will then re-shape these photoresist micro-cylinders into spherical micro-lenses. 


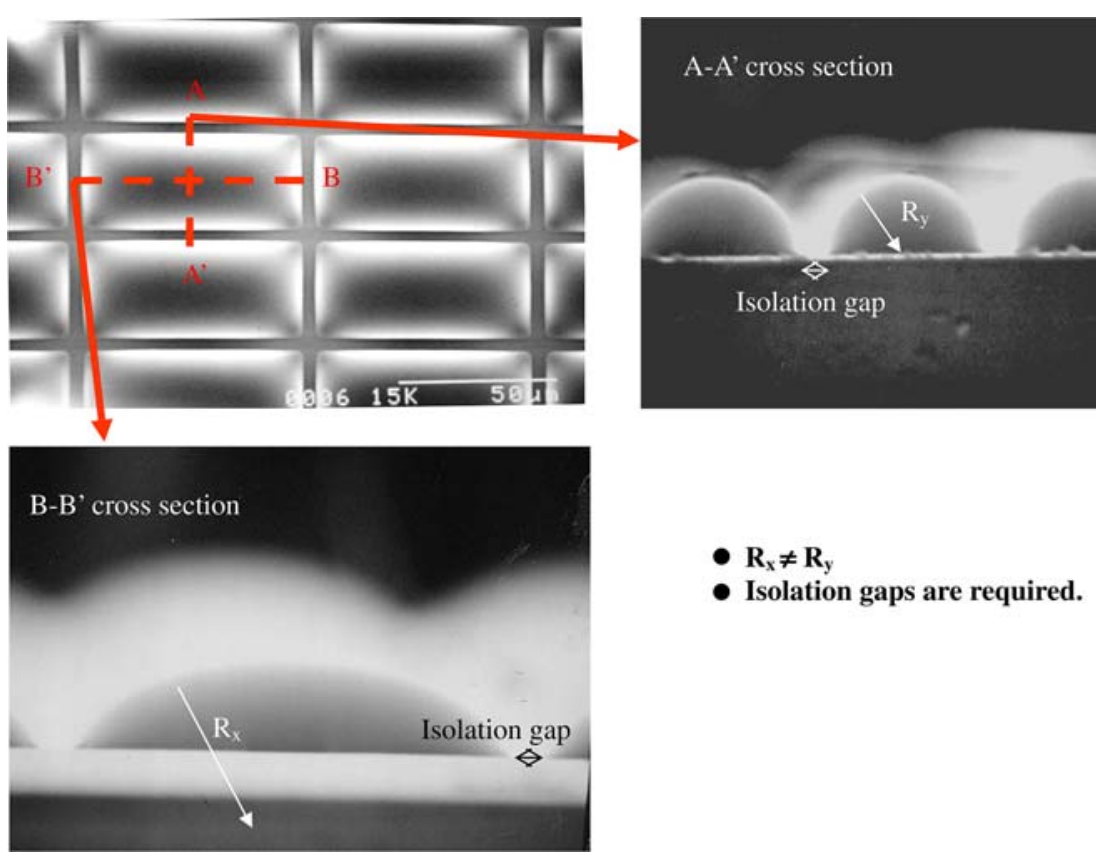

Fig. 1. Typical surface morphology of thermal-reflowed long square lens array.

However, to make surface tension functions properly, small gaps are needed to isolate individual photoresist columns. Hence theoretically it is very difficult if not impossible to produce $100 \%$ filling factor array. There is another drawback of using reflow process. If the initial photoresist pattern is not symmetrical, due to surface tension, it is difficult to obtain micro-lenses with unity curvature of spherical profile. Fig. 1 shows surface profile of thermalrefolwed long square micro-lens. It can be easily observed the isolating gap and different curvatures $\left(R_{x} \neq R_{y}\right)$ at long and short axes. Another method widely adopted process to fabricate multi-level micro-structures to approach refractive lens profile is the binary photolithography $[8,9]$. For a $2^{M}$-level micro-lens array, this method includes $M$ exposures, $(M-1)$ alignments, and $M$ etching steps. Unity curvature can be achieved by designing binary pattern on $M$ masks. Lens gap can also be minimized by reducing feature size of each level. However, to achieve quasi-continuous

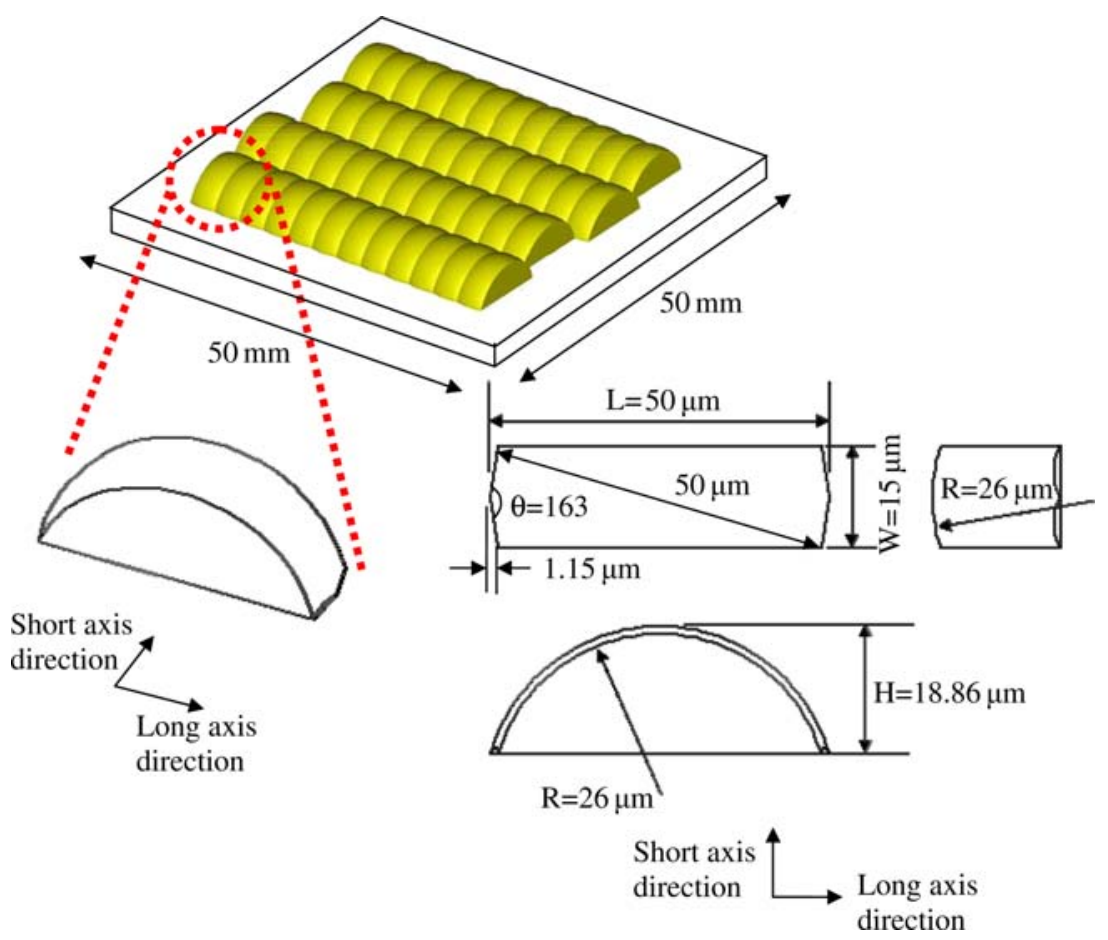

Fig. 2. Design specifications of gapless long HML array. 
surface profile, a number of exposure steps are required and the accumulated errors can easily affect the accuracy of lens profile.

Gray-scale lithography was frequently adopted to allow more phase levels to be produced on the micro-scale/mesoscale optics [10,11]. It contains all the informations required in one gray mask and a continuous surface profile is easily obtained by single exposure. As a result, gray-scale photolithography method can greatly improve the process efficiency by reducing the number of processing steps needed to generate refractive micro-lens profile [9-12]. In applying this method, the high energy beam sensitive (HEBS) mask having high attenuation in the deep ultraviolet spectral range is often employed. Numbers of phase levels are produced on HEBS mask if electron beam lithography is used. The curvature can be controlled by adjusting gray scale levels and the gap between lenses can approach zero ideally.

In this paper, an asymmetrical hexagonal micro-lens (HML) used for light emitting purpose was designed and fabricated. To obtain ideal spherical lens profile and near $100 \%$ filling factor, accurate optical density (OD) was employed in gray-scale lithography process. By applying precision micro-replication process, a high quality HML array was duplicated on flexible polyethylene terphthalate (PET) film. The fabrication quality was inspected and compared with the designed specifications. The optical performance of different light divergent angles of HML was demonstrated.

\section{Specifications of long hexagonal micro-lens array}

To produce different light angle in $x$ - and $y$-directions, the micro-lens is designed in a long asymmetric hexagonal shape and arrayed in close packed form. The geometric parameters are: the lengths of long axis and short axis of the projected shape are $L=50 \mu \mathrm{m}, W=15 \mu \mathrm{m}$, respectively. The length of the other diagonal lines is $50 \mu \mathrm{m}$, the lens height $H=18.86 \mu \mathrm{m}$, the radius of curvature of micro-lens is $R=26 \mu \mathrm{m}$, and the array size is $50 \mathrm{~mm} \times$ $50 \mathrm{~mm}$. The overlap distance of two adjacent lenses is $d=1.15 \mu \mathrm{m}$. The micro-lens material is UV-curable resin having a refractive index of 1.49 . The schematic diagram of the gapless long hexagonal micro-lens array is shown in Fig. 2. The focal length $f$ for the lens can be written as $f=R /(n-1)$ where, in this study, $f \approx 49 \mu \mathrm{m}$.

\section{Micro-replication process}

Three main processes were used in this study to fabricate long hexagonal micro-lens array on a flexible substrate. As shown schematically in Fig. 3. firstly, the photoresist master of hexagonal micro-lens was fabricated using gray tone lithography process. The photoresist master was then transferred onto a Ni mold using micro-electroplaitng process. Finally, with the help of the obtained precision Ni mold, the hexagonal micro-lens structure was replicated onto flexible PET substrate by roller and UV forming process.

\subsection{Photoresist master fabrication}

Photoresist master fabrication is of crucial importance to the subsequent fabrication process. In the experiment, HEBS mask produced by Canyon Materials, Inc. was used to generate lens profile. Precise design of optical density (OD) of gray mask ensures lens profile to achieve ideal design. The OD is related to different thickness and it was characterized by I85B calibration mask. The test pattern has 200 gray levels and each gray level consists of one test pattern of $100 \mu \mathrm{m}$ by $100 \mu \mathrm{m}$ in size. The 200 gray levels represent OD values range from 0.126 to 2 . To start the photolithography process, a hot-dried silicon wafer was spun with a $30-\mu \mathrm{m}$-thick positive photoresist (AZ-4620). It was prebaked in a conventional oven at $95{ }^{\circ} \mathrm{C}$ for $10 \mathrm{~min}$ to remove excess solvent from photoresist. The EVG

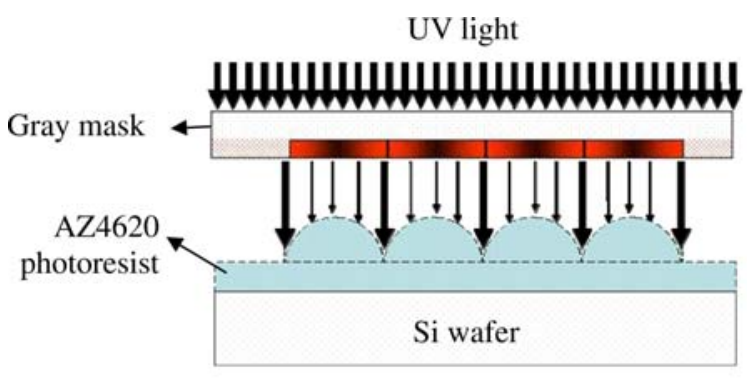

a

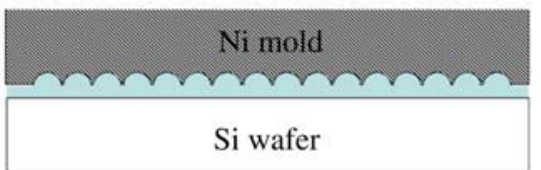

b

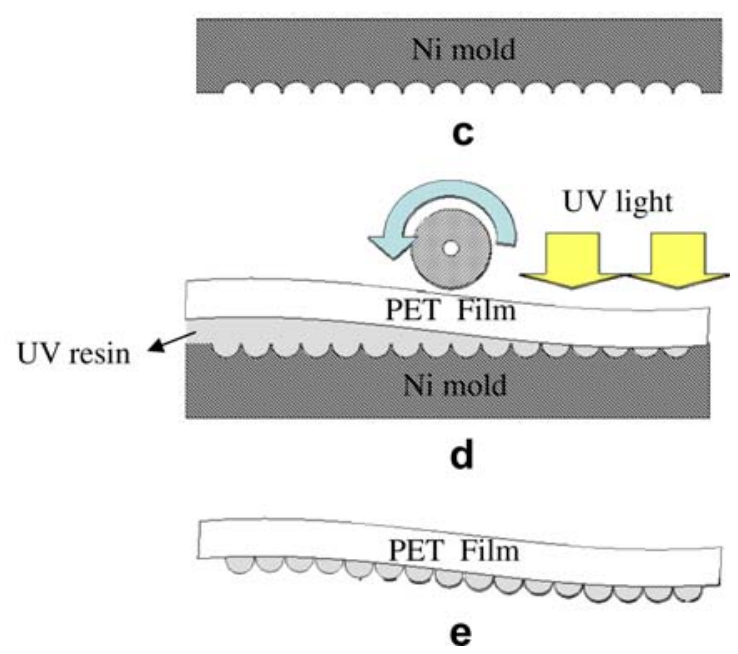

Fig. 3. The schematic flow chart of HML process on flexible PET substrate: (a) gray tone lithography, (b) Ni electroplating, (c) Ni mold demolding, (d) UV casting by a roller on flexible substrate, (e) micro-lens array demolding. 


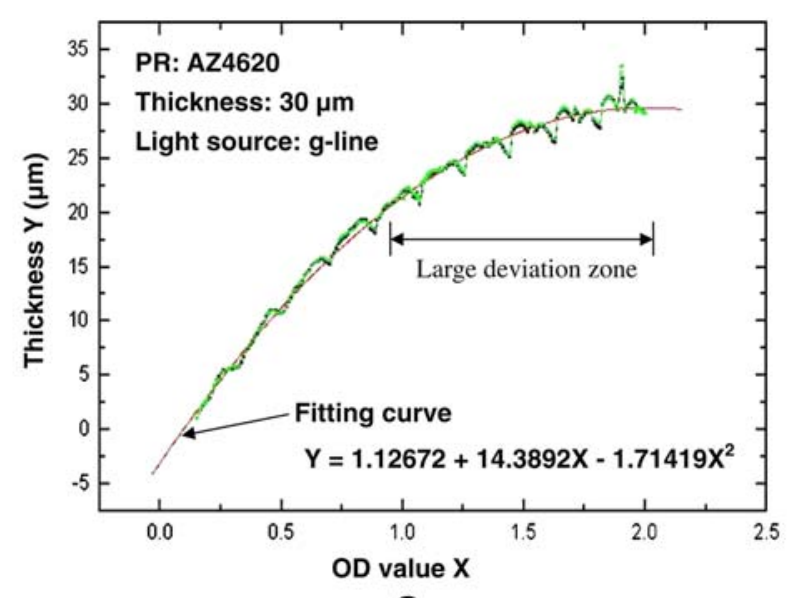

a
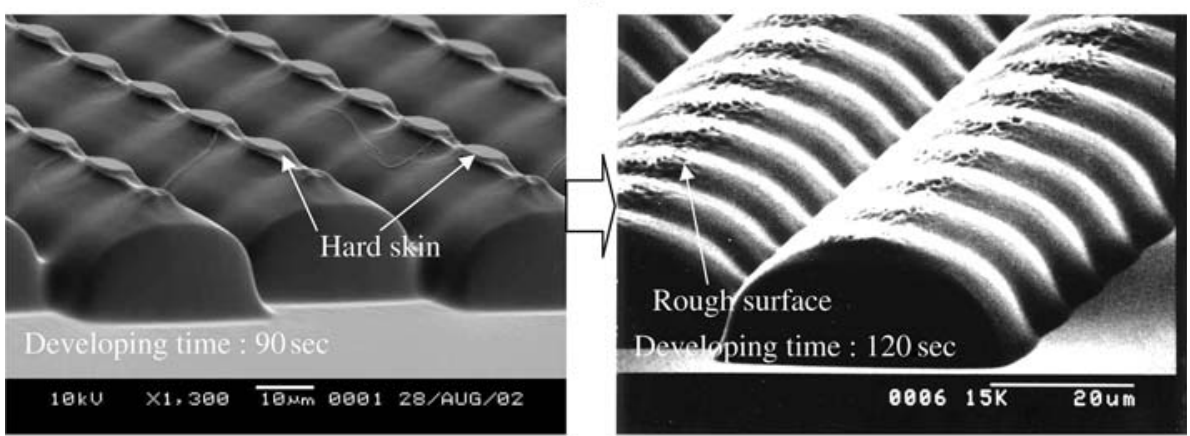

b

Fig. 4. (a) Optical density vs. AZ4620 thickness calibration curve for gray mask, (b) hard skin formed on the top of HML surface profile with gray mask OD range from 0.5 to 2 ; hard skin formed at developing time $90 \mathrm{~s} \mathrm{(left)} \mathrm{and} \mathrm{hard} \mathrm{skin} \mathrm{was} \mathrm{removed} \mathrm{and} \mathrm{rough} \mathrm{surface} \mathrm{formed} \mathrm{on} \mathrm{the} \mathrm{top} \mathrm{at} \mathrm{developing}$ time $120 \mathrm{~s}$ (right).

UV-mask aligner, which has a peak emission at a wavelength of $436 \mathrm{~nm}$ and irradiance of $10 \mathrm{~mW} / \mathrm{cm}^{2}$, was employed for the UV exposure in $80 \mathrm{~s}$. The sample was then dipped in diluted $20 \%$ AZ-400k for $120 \mathrm{~s}$ and rinsed by de-ionized water. The UV irradiation was controlled by the gray level. The higher the OD value the less irradiation could be generated and the thicker photoresist could be obtained. Fig. 4(a) shows the calibration curve of AZ4620 with HEBS gray mask of OD values ranged from 0.126 to 2 . The green ${ }^{3}$ dot line is the real thickness of photoresist with different OD measured by using $\alpha$-step. And the red line is the curve fitting line given by the equation $Y=1.12672+14.3892 X-1.71419 X^{2}$. It is easily seen that the deviation of photoresist thickness from the fitting curve becomes larger when the OD value approaches to 2 . To design OD distribution for accuracy lens shape, a test lens pattern with gray mask OD ranging from 0.5 to 2 was produced to verify the surface quality. In the testing, developing process was interrupted at 90th sec, and a hard skin layer on the top of HML was observed by SEM as shown in Fig. 4(b) left. After full developing condition (developing

\footnotetext{
${ }^{3}$ For interpretation of color in all the figures, the reader is referred to the web version of this article.
}

time $=120 \mathrm{~s}$ ), the hard skin was removed but the surface roughness on the top of HML becomes poor as shown in Fig. 4(b) right. The hard skin is formed on the top of the photoresist layer during prebaking process and it results in the deviation in thickness due to insensitivity of the hard skin layer to the slight change of OD value. To make a smooth surface, lowering OD value is a good choice to keep the top surface of HML away from the hard skin

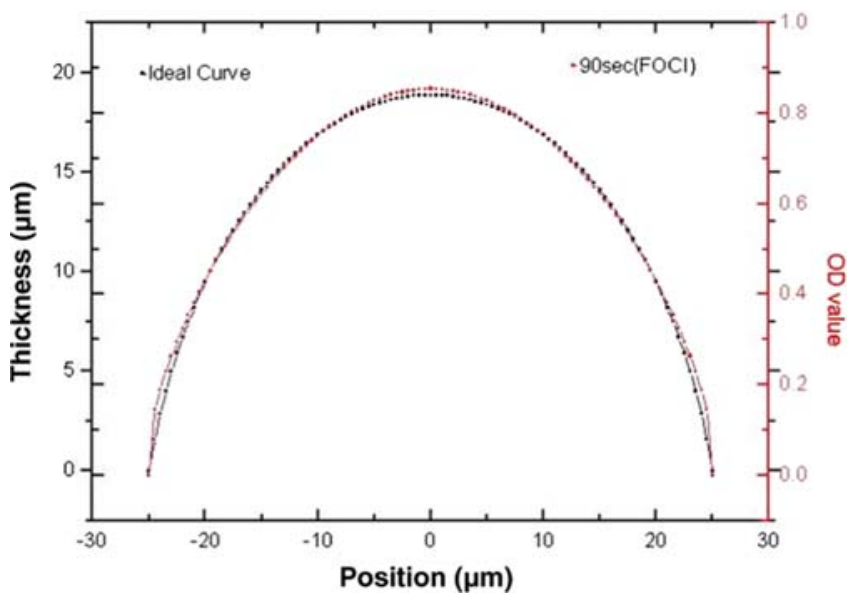

Fig. 5. Designed optical density distribution for HML. 
layer. In this study, OD values ranging from 0.12 to 1 were selected to design micro-lens. The OD distribution for the designed micro-lens is shown in Fig. 5. Many $1-\mu \mathrm{m}$-width rings with the same gray level were used to produce spherical surface. After lithography process, long HML array was completed. Fig. 6(b)-(d) are SEM micro-photographs in different view angles. From the SEM results, the shape of photoresist HML is very close to the ideal profile showing in Fig. 2.

\subsection{Ni mold insert fabrication}

A micro-electroplating process was applied to transfer the photoresist hexagonal micro-lens array onto the $\mathrm{Ni}$ mold. The metallization process included sputtering $100 \mathrm{~nm}$ Ni layer, electroplating $\mathrm{Ni}$, backside lapping and mold releasing. The experimental result showed that the photoresist hexagonal micro-lens mold could be precisely transferred to the Ni mold. The Ni mold has very high duplicability of photoresist micro-structure and has potential for mass production.

\subsection{UV imprinting process}

A modified imprinting process using roller was proposed and implemented in the experiment. The imprinting steps can be summarized as follow ([referring to Fig. 3(d)]): (1) The UV-curable material in liquid form was dispensed onto the Ni template to fill concave cavity of HML. For good demolding property, a flexible PET film as HML substrate was bent and placed over the dispensed resin to avoid any air bubbles being trapped in the resin layer.
The PET film is transparent to allow the following UV irradiation. (2) The coated template with PET film was laminated by a height controlled roller and it moved from one side to the other to remove redundant resin and controlled the UV resin layer thickness. The $200-\mu \mathrm{m}$-thick coated resin layer was obtained. (3) The sample was exposed in the curing system for $12 \mathrm{~min}$ and then the resin was solidified at room temperature. The general UV light of the wavelength of $250-420 \mathrm{~nm}$ was used in the experiment and the exposure intensity was $40 \mathrm{~mW} / \mathrm{cm}^{2}$ which was measured by photometer at $365 \mathrm{~nm}$ wavelength. (4) After curing step, the flexible PET film with HML structure can be pealed off from HML template. The SEM image of prototype is shown in Fig. 6. Gapless long HML structure was duplicated successfully and no significant damage was found. Hence, good replication quality of UV imprinting with flexible substrate was demonstrated. The SEM images of the obtained HML structure in different view angles are displayed in Fig. 6.

\section{Measurement results and discussion}

To analyze fabrication quality, geometry characters of HML structure was inspected to compare with designed values. To measure the lengths of long axis $L$ and short axis $W$ of the projected shape, HML was observed by using $500 \mathrm{X}$ optical microscope and the length and width were directly read out from micrometer on the movable stage [Fig. 7(a)]. The lens height $H$, curvature of lens at long axis $R_{\mathrm{L}}$ and at short axis $R_{\mathrm{S}}$ were evaluated using SEM observation. In the cross-section view of SEM micrograph, the lens height was directly measured by using a scale bar.

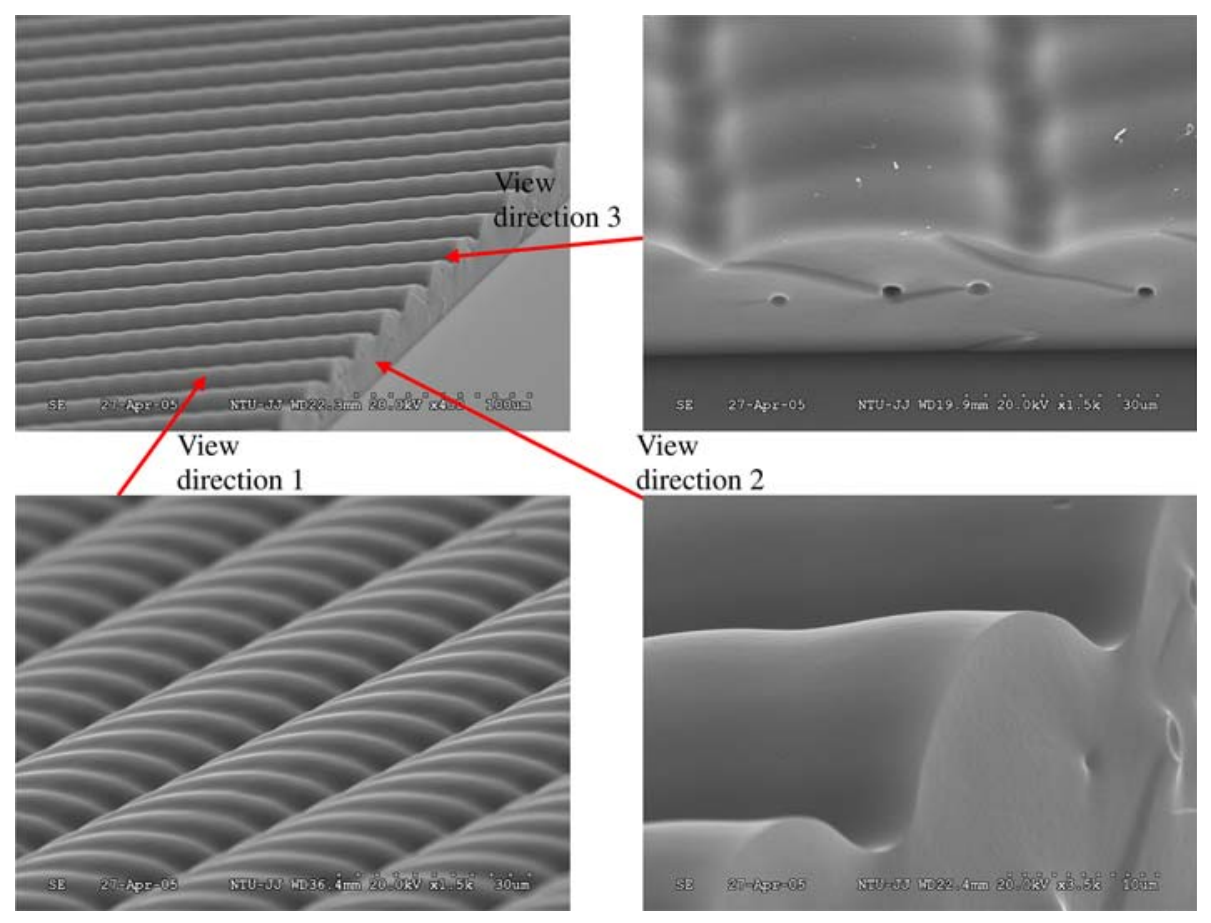

Fig. 6. SEM images of UV casting micro-lens structures on flexible PET substrate and the local area. 


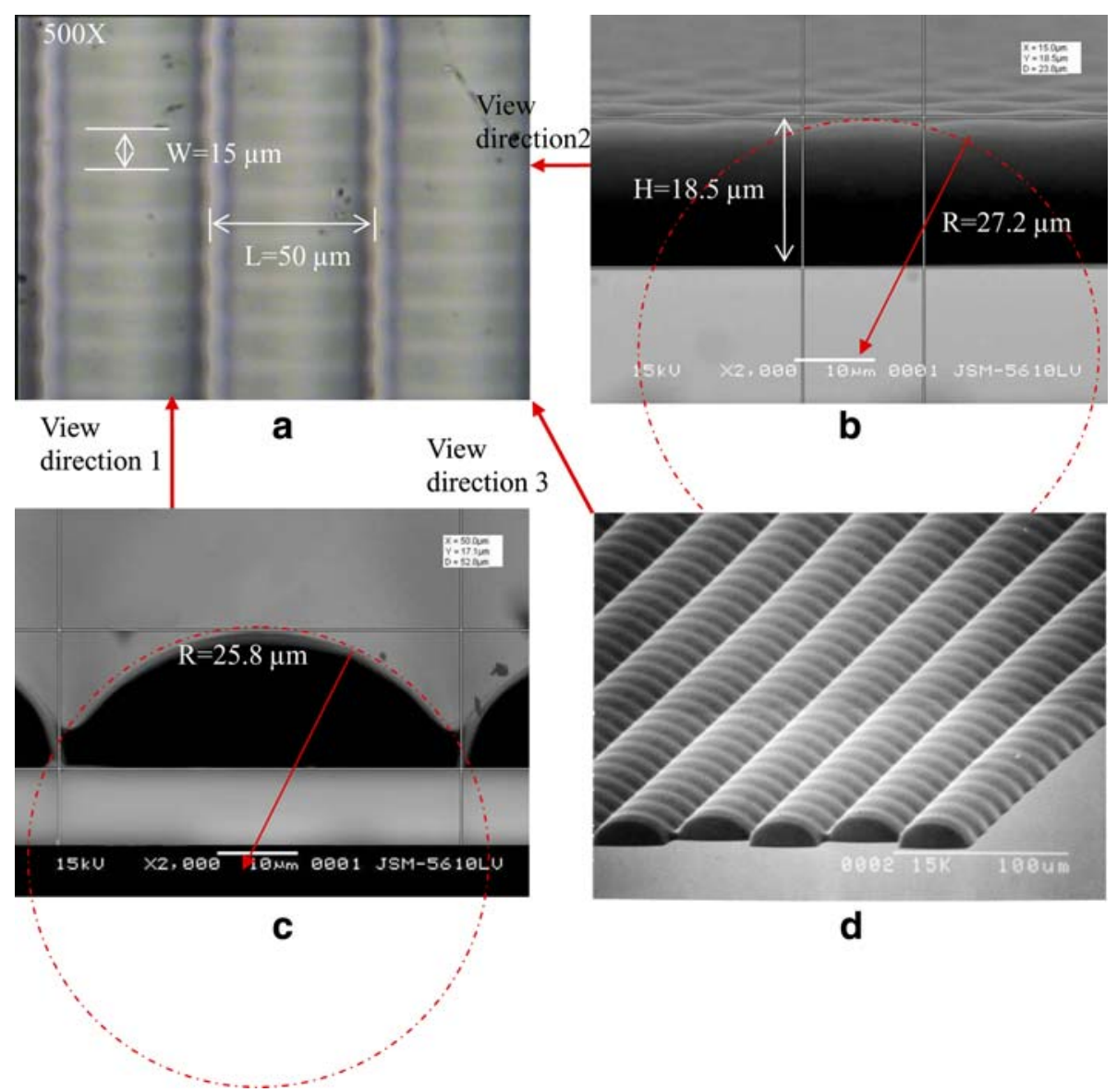

Fig. 7. Geometry inspection of AZ4620 HML (a) 500X optical microscope image to inspect the length of HML at long and short axis. (b) SEM crosssection image of HML at short axis to inspect radius of curvature and lens height. (c) SEM cross-section image of HML at long axis to inspect radius of curvature. (d) SEM image of another view angle.

The radius of curvature of lens was evaluated by fitting proper radius of circle with lens profile at both long and short axes [Fig. 7(b) and (c)]. The filling factor of HML was defined as ratio of actual lens area to the designed lens area. That is,

Filling factor $\equiv\left|\frac{\text { measurement lens area } A^{\prime}}{\text { designed lens area } A}\right|$

$$
=\frac{W^{\prime} \times\left\{L^{\prime}-\frac{W^{\prime}}{2} \cot \left(\frac{\theta}{2}\right)\right\}}{W \times\left\{L-\frac{W}{2} \cot \left(\frac{\theta}{2}\right)\right\}}
$$

The filling factor can be calculated by substituting $L, W$, $L^{\prime}, W^{\prime}$ and $\theta$ into the above expression.

The measured results of geometry characters of photoresist master and UV-replicated structure are listed in Table 1. For photoresist master, the lengths at long and short axis direction are about $50 \mu \mathrm{m}$ and $14.8 \mu \mathrm{m}$, respectively; the height of HML is about $18.5 \mu \mathrm{m}$ and the radii of curvature at long and short axis directions are about $25.8 \mu \mathrm{m}$ and $27.2 \mu \mathrm{m}$, respectively. Based on these results, a high-quality photoresist master with good dimension control during fabrication and near unity curvature was successfully fabricated. For the UV-replicated structure, the dimensional error increased slightly due to two pattern transfers of $\mathrm{Ni}$ mold and UV-replication. The lengths of HML at long and short axis directions are about $49 \mu \mathrm{m}$ and $14.6 \mu \mathrm{m}$, respectively; the height of HML is reduced to $14.7 \mu \mathrm{m}$; the radii of curvature at long and short axis directions are about $27.3 \mu \mathrm{m}$ and $26.4 \mu \mathrm{m}$. To evaluate the surface condition affected by replication, the surface roughness $\left(R_{\mathrm{a}}\right)$ of HML was measured by AFM. The $R_{\mathrm{a}}$ at the top of photoresist and UV-replicated HML were found around $17 \mathrm{~nm}$ and $28 \mathrm{~nm}$, respectively.

Table 1

Measurement results of fabrication quality

\begin{tabular}{llll}
\hline Item & Design value & \multicolumn{2}{l}{ Measurement value } \\
\cline { 3 - 4 } & & 50 & 49 \\
\hline $\begin{array}{l}\text { Length of projected } \\
\text { shape at long axis, } L(\mu \mathrm{m})\end{array}$ & 50 & & \\
$\begin{array}{l}\text { Length of projected } \\
\text { shape at short axis, } W(\mu \mathrm{m})\end{array}$ & 15 & 14.8 & 14.6 \\
$\quad$ Lens height, $H(\mu \mathrm{m})$ & 18.86 & 18.5 & 14.7 \\
$\quad$ Radius of curvature & 26 & 25.8 & 27.3 \\
$\quad$ at long axis, $R_{\mathrm{L}}(\mu \mathrm{m})$ & & & \\
Radius of curvature & & 27.2 & 26.4 \\
$\quad$ at short axis, $R_{\mathrm{S}}(\mu \mathrm{m})$ & & 17 & 28 \\
$\quad \begin{array}{l}\text { Surface roughness, } R_{\mathrm{a}}(\mathrm{nm}) \\
\text { Filling factor }(\%)\end{array}$ & & 98.7 & 95.4 \\
\hline
\end{tabular}




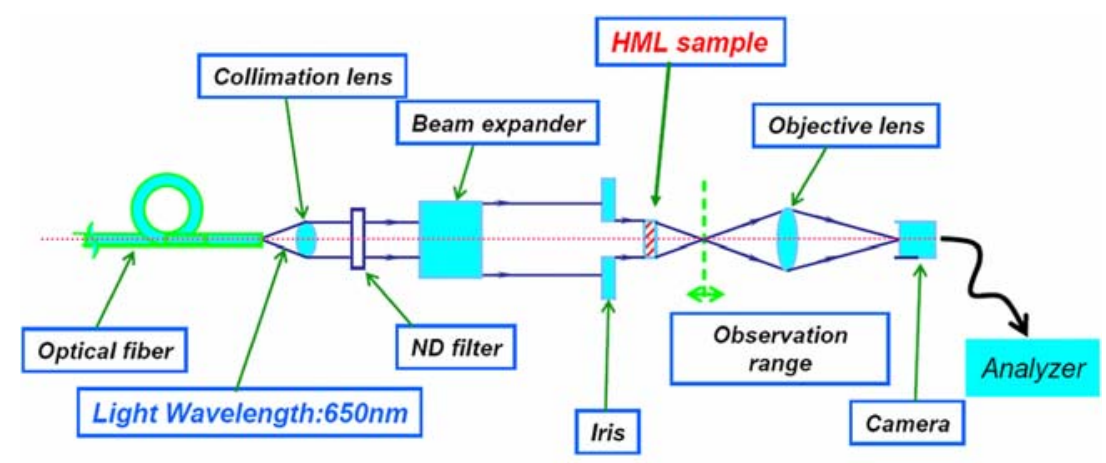

Fig. 8. Schematic illustration of the measurement setup.

To inspect the optical properties of HML, the measurement setup shown in Fig. 8 was constructed. Here image acquisition (camera) and analysis (analyzer) were performed with a Hamamatsu Lepas 11 system and a dedicated optics and camera as well. The symmetry Gauss beam was used in the experiment and its wavelength is $650 \mathrm{~nm}$. The measurement results of optical beam profile of gapless long hexagonal micro-lens in the focal plane are shown in Fig. 9 and Table 2. Fig. 9(a)-(c) are measured image, three-dimensional display and optical intensity profiles for long axis and short axis of HML, respectively. One can see that the measured profile is obviously asymmetrical. The beam full widths at the half maximum (FWHM) in short axis and long axis directions through the long

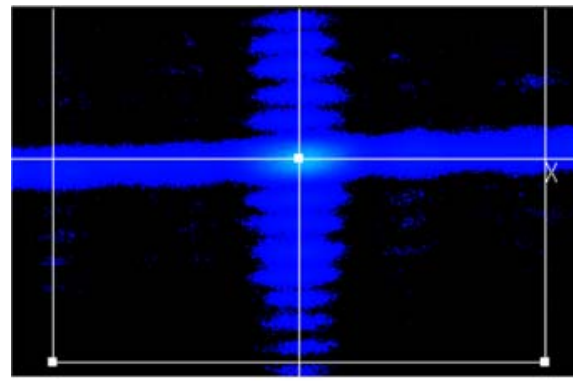

a

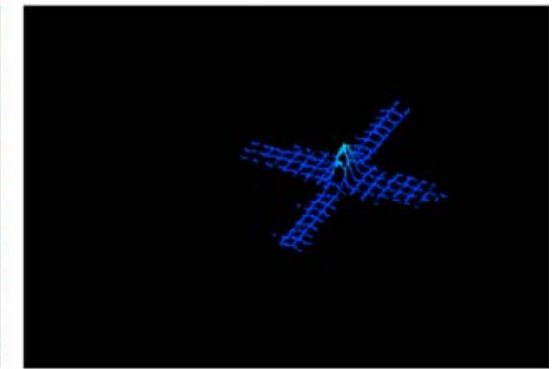

b
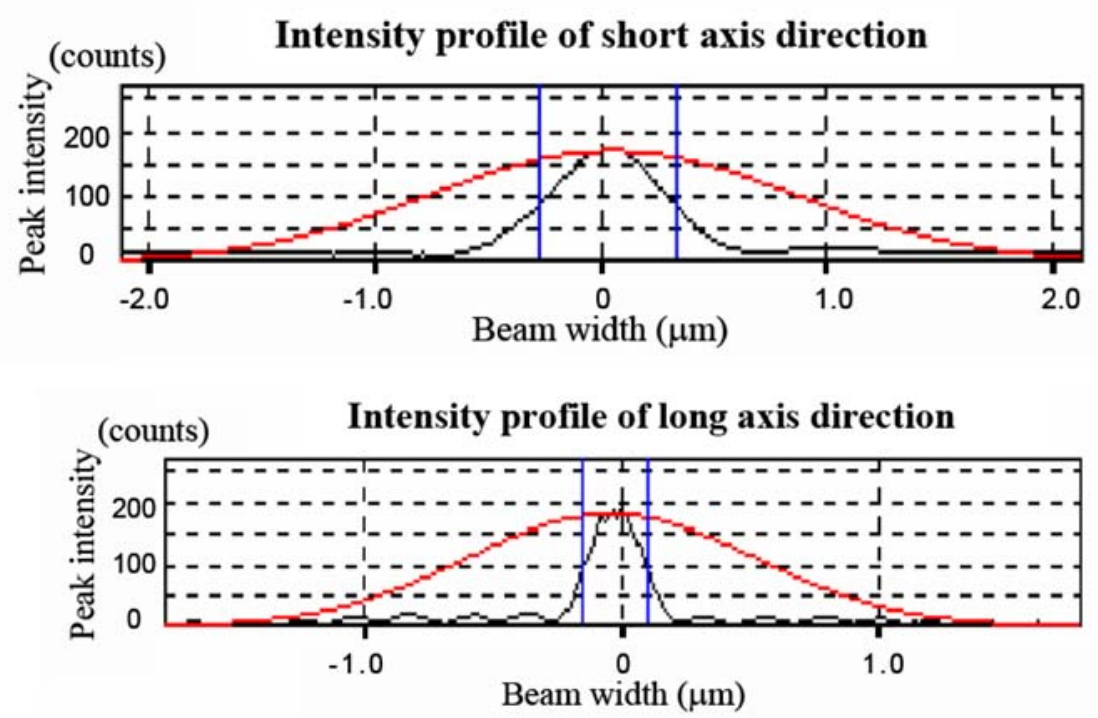

Input Gauss beam profile
Output beam profile

C

Fig. 9. Measurement results of optical beam profile of gapless long HML in the focal plane. (a) Measured image, (b) three-dimensional display and (c) optical intensity profiles for long axis and short axis of HML. 
Table 2

Measurement results of optical beam profile on the focal plane

\begin{tabular}{llll}
\hline Item & & $\begin{array}{l}\text { Input Gauss } \\
\text { beam width }(\mu \mathrm{m})\end{array}$ & $\begin{array}{l}\text { Output beam } \\
\text { width }(\mu \mathrm{m})\end{array}$ \\
\hline FWHM & Short axis & 1.90 & 0.61 \\
& Long axis & 1.33 & 0.25 \\
$1 / \mathrm{e}$ & Short axis & 2.29 & 0.74 \\
& Long axis & 1.59 & 0.3 \\
$1 / \mathrm{e}^{2}$ & Short axis & 1.08 & 0.39 \\
& Long axis & 3.23 & 2.24 \\
\hline
\end{tabular}

HML are $0.61 \mu \mathrm{m}$ and $0.25 \mu \mathrm{m}$, respectively. The beam full widths at the $1 / \mathrm{e}^{2}$ intensity in short axis and long axis directions through the long HML are $1.08 \mu \mathrm{m}$ and $0.39 \mu \mathrm{m}$, respectively. Hence, the beam divergent ratio at long and short axis of 2.44:1 was achieved.

\section{Conclusion}

A long HML array was designed to generate different light divergent angles in $x$ - and $y$-directions. Gray-scale lithography was used to achieve ideal spherical lens and near $100 \%$ filling factor. The prototype of HML was successfully produced by Micro-replication process. The geometry dimensions of the obtained photoresist HML are $L \approx 50 \mu \mathrm{m}, W \approx 15 \mu \mathrm{m}, H \approx 18.5 \mu \mathrm{m}$ and they are very close to the targeted specifications. Near unity curvature $\left(R_{\mathrm{L}} \approx 25.8 \mu \mathrm{m}, R_{\mathrm{S}} \approx 27.2 \mu \mathrm{m}\right)$ and filling factor of $98.7 \%$ were achieved. Based on the results of the optical experiment, the incident Gauss beam was successfully diversified differently at short and long axis. The beam FWHM at short axis and long axis directions through the long HML are $0.61 \mu \mathrm{m}$ and $0.25 \mu \mathrm{m}$, respectively. Hence, the beam divergent ratio at long and short axis of 2.44:1 was achieved.

\section{Acknowledgements}

The authors would like to thank Dr. T.C. $\mathrm{Wu}, \mathrm{Mr}$. M.Y. Liu, Mr. C.K. Mu and J.S. Tsai at MIRL of ITRI, Taiwan for their guidance and assistance.

\section{References}

[1] N.F. Borrelli, Microoptics Technology, Marcel Dekker, New York, 1999.

[2] S. Sinzinger, J. Jahns, Microoptics, second ed., Wiley-VCH, Weinheim, 2003.

[3] R.H. Anderson, Appl. Opt. 18 (1979) 477.

[4] M.E. Motamedi, Opt. Eng. 33 (1994) 3505.

[5] C. Berger, N. Collings, R. Vökel, M.T. Gale, T. Hessler, Pure Appl. Opt. 6 (1997) 683.

[6] F.B. McCormick, F.A.P. Tooley, T.J. Cloonan, J.M. Sasian, H.S. Hinton, Opt. Quantum Electron. 24 (1992) S465.

[7] M.C. Hutley, Microlens Array, IOP Publishing Ltd, 1991.

[8] J.J. Yang, Y.S. Liao, C.F. Chen, Jpn. J. Appl. Phys. 45 (2004) 2812.

[9] W. Daschner, P. Long, R. Stein, C. Wu, S.H. Lee, Appl. Opt. 36 (1997) 4675.

[10] Z. Zhou, S.H. Lee, Opt. Lett. 29 (2004) 457.

[11] X.C. Yuan, W.X. Yu, N.Q. Ngo, W.C. Cheong, Opt. Express 10 (2002) 303.

[12] J.S. Sohn, M.B. Lee, W.C. Kim, E.H. Cho, T.W. Kim, C.Y. Yoon, N.C. Park, Y.P. Park, Appl. Opt. 44 (2005) 506. 